\title{
Detecting light-induced Floquet band gaps of graphene via trARPES
}

\author{
Lukas Broers $\oplus^{1,2}$ and Ludwig Mathey ${ }^{1,2,3}$ \\ ${ }^{1}$ Center for Optical Quantum Technologies, University of Hamburg, 22761 Hamburg, Germany \\ ${ }^{2}$ Institute for Laser Physics, University of Hamburg, 22761 Hamburg, Germany \\ ${ }^{3}$ The Hamburg Center for Ultrafast Imaging, Luruper Chaussee 149, 22761 Hamburg, Germany
}

(Received 12 August 2021; revised 21 December 2021; accepted 23 December 2021; published 28 January 2022)

\begin{abstract}
We propose a realistic regime to detect the light-induced topological band gap in graphene via time-resolved angle-resolved photoelectron spectroscopy (trARPES), which can be achieved with current technology. The direct observation of Floquet-Bloch bands in graphene is limited by low-mobility, Fourier-broadening, laserassisted photoemission (LAPE), probe-pulse energy-resolution bounds, space-charge effects, and more. We characterize a regime of low driving frequency and high amplitude of the circularly polarized light that induces an effective band gap at the Dirac point that exceeds the Floquet zone. This circumvents limitations due to energy resolutions and band broadening. The electron distribution across the Floquet replicas in this limit allows for distinguishing LAPE replicas from Floquet replicas. We derive our results from a dissipative master equation approach that gives access to two-point correlation functions and the electron distribution relevant for trARPES measurements.
\end{abstract}

DOI: 10.1103/PhysRevResearch.4.013057

\section{INTRODUCTION}

Floquet engineering constitutes a novel approach to control material properties via light [1-4]. A prominent example is the proposed light-induced topologically insulating state of monolayer graphene [5-7]. The resulting anomalous Hall effect in this system has been observed experimentally [8] and has been explained as a geometric-dissipative effect [9] in accordance with Floquet theory. Meanwhile, time-resolved angle-resolved photoemission spectroscopy (trARPES) has been established as the prime method for resolving dynamical changes in effective band structures of solid-state systems [10-16]. Experimental trARPES setups are constantly improving and being used for investigating the dynamical electronic processes in two-dimensional Dirac materials such as graphene [17-21], $\mathrm{WSe}_{2}$ [20-24], and $\mathrm{Bi}_{2} \mathrm{Se}_{3}$ [25-28]. Approaches related to observing pseudospin textures in ARPES were discussed in Refs. [29-32]. In $\mathrm{Bi}_{2} \mathrm{Se}_{3}$, Floquet replicas of electronic bands were observed using trARPES setups [33]. However, the direct observation of both the replicas and the topological gap at the Dirac point has been met with intricate challenges in graphene and remains unachieved to date.

In this work we determine the regime of trARPES measurements for observing the topological band gap at the Dirac point of irradiated graphene. We propose to perform these measurements in the regime of low driving frequencies and high driving field strengths. In this previously unexplored

Published by the American Physical Society under the terms of the Creative Commons Attribution 4.0 International license. Further distribution of this work must maintain attribution to the author(s) and the published article's title, journal citation, and DOI. regime the dominant Floquet-Bloch band occupations are spaced farther apart than the driving frequency. They are therefore outside of the first Floquet zone. We propose to detect this light-induced energy gap beyond the Floquet zone in experiment because the different spectral features are well resolved in this regime. We discuss the dependence of our predictions on the system parameters, specifically how they affect the systematic limitations of the energy resolution of photoemission spectroscopy. These parameters include the driving frequency and field strength, which determine the Floquet-Bloch band structure, the dissipation coefficients that broaden the band signals, and the pulse lengths of drive and probe lasers. For the pulse lengths we point out a desirable regime with sufficient energy resolution and high enough repetition rates. These repetition rates are necessary to avoid undesired space-charge effects, where the photoemitted electrons interact and affect each other's trajectories [23,3436]. In particular, it is possible to distinguish the laser-assisted photoemission (LAPE) replicas from the Floquet replicas within the gap at the Dirac point in our suggested regime.

We consider a single layer of graphene irradiated by a circularly polarized infrared laser from a perpendicular direction. We consider a laser pulse with a temporal Gaussian envelope of pulse length $\tau_{\mathrm{d}}$. The pulse length is assumed to be much longer than the driving period, so that it induces Floquet-Bloch states that vary with the envelope function of the pulse. The graphene sample is probed by a tunable extreme ultraviolet laser pulse from the same direction. It has a shorter pulse length and excites photoelectrons out of the driven graphene over a time span during which the driving intensity is approximately constant. This is necessary for resolving the time-dependent Floquet-Bloch bands, which are sensitive to the driving amplitude. This is considered the standard approach to trARPES experiments [37] and is illustrated 


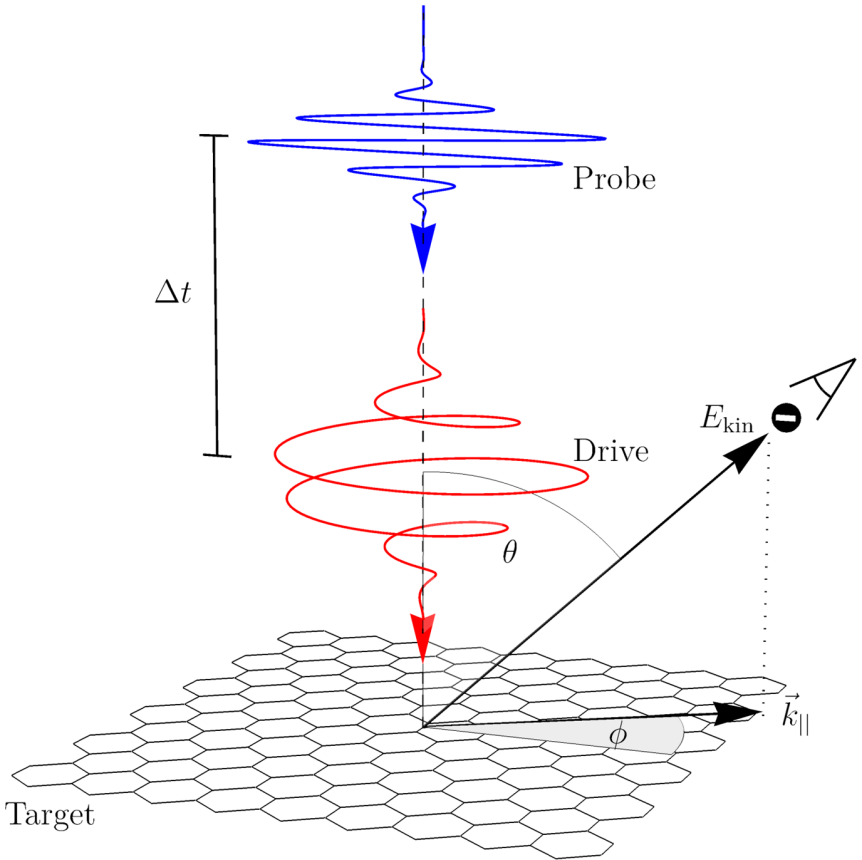

FIG. 1. An illustration of the trARPES principle. A circularly polarized infrared drive pulse (red) hits the graphene target from a perpendicular direction, exciting a transient state in the illuminated region. After a time delay of $\Delta t$, an ultraviolet probe pulse (blue) hits the target and excites photoelectrons. A given electron leaves the target with a kinetic energy $E_{\text {kin }}$ at an inclination $\theta$ to the graphene target and at an azimuthal angle $\phi$. The momentum component $\vec{k}_{\|}$ parallel to the target is that of the preprobe transient state of the electron bound in the graphene layer.

in Fig. 1. The emitted photoelectrons corresponding to a probe frequency $\omega_{\mathrm{p}}$ have the kinetic energy

$$
E_{\mathrm{kin}}=\hbar \omega_{\mathrm{p}}-E_{\mathrm{b}}-\Phi,
$$

where $E_{\mathrm{b}}$ is the binding energy and $\Phi$ is the work function of the material, which is the energy required to remove the electron from the graphene. In addition, a photoelectron has momentum $\vec{k}$ with components

$$
\vec{k}_{\|}=\sqrt{2 m E_{\mathrm{kin}}} \hbar^{-1} \sin (\theta)(\cos (\phi), \sin (\phi))^{\mathrm{T}}
$$

parallel to the graphene layer. $m$ is the electron mass, and $\theta$ and $\phi$ are the inclination and azimuthal angles of the momentum with respect to the graphene layer. Measuring the photoelectron counts at these angles and energies gives access to the time-resolved Floquet-Bloch bands. The momentum $\vec{k}_{||}$ is the electron momentum prior to the excitation process. For simplicity this is denoted as $\mathbf{k}$ in the following.

\section{PREDICTIONS FOR trARPES MEASUREMENTS}

We describe the electron dynamics in graphene with the Hamiltonian

$$
H(t)=\sum_{\mathbf{k}} c_{\mathbf{k}}^{\dagger} h(\mathbf{k}, t) c_{\mathbf{k}}
$$

where $c_{\mathbf{k}}=\left(c_{\mathbf{k}, A}, c_{\mathbf{k}, B}\right)^{\mathrm{T}}$. Here $c_{\mathbf{k}, i}^{(\dagger)}$, with $i \in\{A, B\}$, are the annihilation (creation) operators of the graphene sublattices.
The single-particle Hamiltonian is given by

$$
h(\mathbf{k}, t)=\hbar v_{F}\left[q_{x}(t) \sigma_{x}+q_{y}(t) \sigma_{y}\right],
$$

with

$$
\begin{aligned}
& q_{x}(t)=k_{x}+\frac{e}{\hbar} A_{\mathrm{d}}(t) \sin \left(\omega_{\mathrm{d}} t\right), \\
& q_{y}(t)=k_{y}+\frac{e}{\hbar} A_{\mathrm{d}}(t) \cos \left(\omega_{\mathrm{d}} t\right),
\end{aligned}
$$

where $v_{F} \approx 10^{6} \mathrm{~ms}^{-1}$ is the Fermi velocity and $k_{x}=$ $|\mathbf{k}| \cos (\phi)$ and $k_{y}=|\mathbf{k}| \sin (\phi)$ are the momentum components. $\sigma_{i}$ are the Pauli matrices. The pulsed vector potential amplitude is given by

$$
A_{\mathrm{d}}(t)=\frac{E_{\mathrm{d}}}{\omega_{\mathrm{d}}} \exp \left\{-t^{2} \tau_{\mathrm{d}}^{-2} 4 \ln (2)\right\},
$$

where $\tau_{\mathrm{d}}$ is the driving pulse full width at half maximum (FWHM). For the pulse length we use the value $\tau_{\mathrm{d}}=500 \mathrm{fs}$ as a realistic value for driving frequencies in the range of tens to hundreds of terahertz. Note that the specific choice of a temporal Gaussian envelope is not crucial for the results. However, more quickly varying temporal envelopes may induce transient behavior that potentially obscure band signals.

We consider a product state $\rho=\Pi_{\mathbf{k}} \rho_{\mathbf{k}}$ and evolve the system using a Lindblad-von Neumann master equation that includes dissipation. The dissipation channels amount to dephasing, decay, and electron exchange with a back gate. The corresponding coefficients are chosen to be $\gamma_{z}=22.5 \mathrm{THz} \approx$ $(44.4 \mathrm{fs})^{-1}, \gamma_{ \pm}=10 \mathrm{THz}=(100 \mathrm{fs})^{-1}$ and $\gamma_{\mathrm{bg}}=25 \mathrm{THz}=$ $(40 \mathrm{fs})^{-1}$. These are the values that were demonstrated to describe the experimental realization of Ref. [8] in Ref. [9]. Hence, this approach is well suited to describing the dynamics in realistic solid-state samples, despite the potential intricacies of material defects [38-41]. These values for the dissipation coefficients also agree with the relaxation times of 20 to $40 \mathrm{fs}$ found in Refs. [42-45] and the electron-phonon channel relaxation estimated to be on the order of $100 \mathrm{fs}$ [44-46]. This also gives the timescale of the system to form a steady state with an effective Floquet spectrum. We include a nonzero temperature in the system by giving corresponding Boltzmann factors to complementary dissipation coefficients so that the equilibrium states realize the desired temperature $T$. Throughout this work we use room temperature $T=300 \mathrm{~K}$. For details of this approach, see Ref. [9] or Appendix A.

Our predictions for the trARPES measurements are based on the momentum- and energy-resolved electron distribution calculated as [47]

$$
n(k, \omega)=\int_{-t_{0}}^{t_{0}} \int_{-t_{0}}^{t_{0}} s\left(t_{1}\right) s\left(t_{2}\right) \mathcal{G}\left(k, t_{2}, t_{1}\right) \frac{e^{i \omega\left(t_{2}-t_{1}\right)}}{4 t_{0}^{2}} d t_{2} d t_{1}
$$

with the correlator [48]

$$
\mathcal{G}\left(\mathbf{k}, t_{2}, t_{1}\right)=\sum_{i \in\{A, B\}}\left\langle c_{\mathbf{k}, i}^{\dagger}\left(t_{2}\right) c_{\mathbf{k}, i}\left(t_{1}\right)\right\rangle
$$

and the probe pulse envelope

$$
s(t)=\exp \left\{-(t-\Delta t)^{2} \tau_{\mathrm{p}}^{-2} 4 \ln (2)\right\},
$$

where $\tau_{\mathrm{p}}$ is the probe pulse length (FWHM) and $\Delta t$ is the delay time between the incidence of the drive and probe pulses. For the probe length we use the value $\tau_{\mathrm{p}}=100 \mathrm{fs}$. $t_{0}$ is the 
(a)

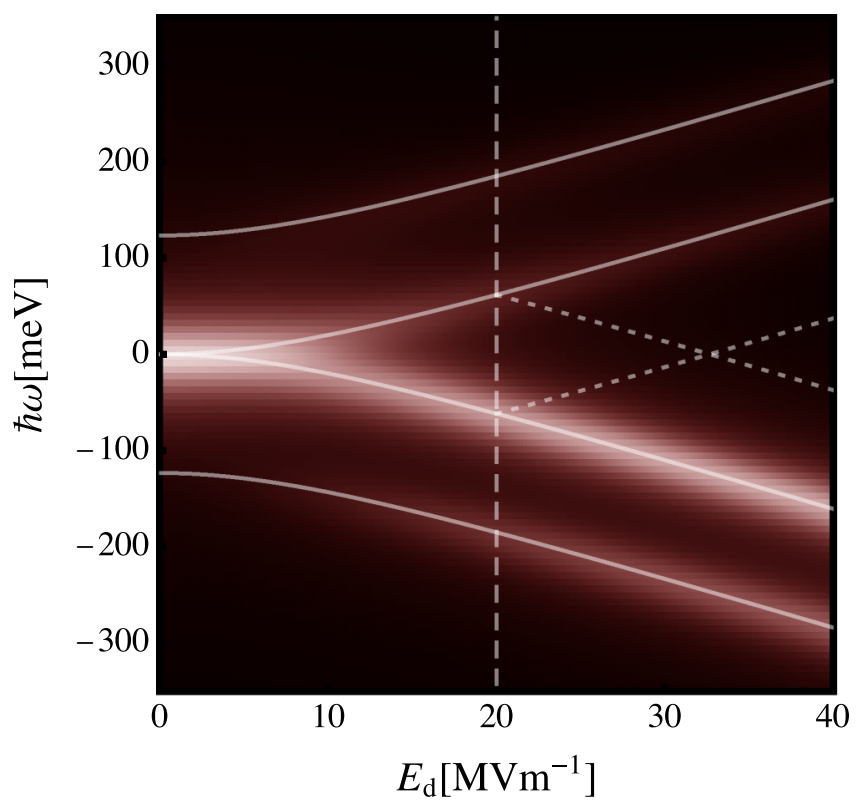

(b)

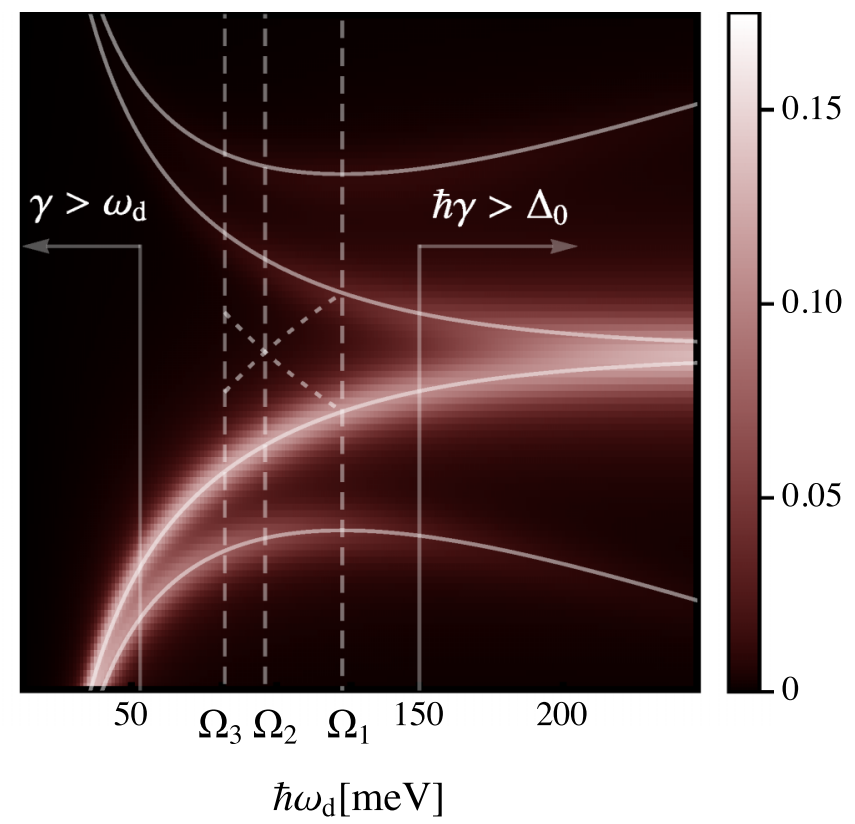

FIG. 2. The electron distribution at the Dirac point $n(\mathbf{k}=0, \omega)$ for zero delay $(\Delta t=0)$ (a) as a function of the driving field strength at the driving frequency $\omega_{\mathrm{d}}=2 \pi \times 29.8 \mathrm{THz} \approx 123 \mathrm{meV} \hbar^{-1}$ and (b) as a function of the driving frequency at driving field strength $E_{\mathrm{d}}=$ $20 \mathrm{MV} \mathrm{m}^{-1}$. The solid lines show the expected Floquet energies $\pm \sqrt{\left(e v_{F} E_{\mathrm{d}} / \omega_{\mathrm{d}}\right)^{2}+\left(\hbar \omega_{\mathrm{d}} / 2\right)^{2}} \pm \hbar \omega_{\mathrm{d}} / 2$. The dotted lines show the Floquet energies confined within the first Floquet zone of width $\hbar \omega_{\mathrm{d}}$. The dashed lines indicate the field strength at which $\Delta_{0}=\hbar \omega_{\mathrm{d}}$ in (a) and the driving frequencies $\Omega_{m=1,2,3}$ in (b). The frequencies $\Omega_{m=1,2,3}$ are defined in Eq. (14). The examples shown in Figs. 3 and 4 also use these frequencies.

temporal integration range, for which we choose $t_{0}=3 \tau_{\mathrm{d}}$ to support the probe pulse sufficiently well.

We choose the pulse lengths of the drive and the pump pulse to fulfill two requirements. First, the probe pulse length is chosen to be short compared to the drive pulse length, so that the drive-induced dynamics is resolved. Second, the probe pulse length is chosen to be large compared to the driving period. These conditions are expressed as

$$
\tau_{\mathrm{d}} \gg \tau_{\mathrm{p}} \gg \frac{2 \pi}{\omega_{\mathrm{d}}} .
$$

When the probe pulse length $\tau_{\mathrm{p}}$ and the driving period $2 \pi / \omega_{\mathrm{d}}$ are comparable, Eq. (8) no longer resolves Floquet-Bloch bands but rather resolves subdriving period electron occupations. We note that increasing the pulse lengths requires increasing the drive pulse energies, which are experimentally limited. This also leads to a reduced repetition rate, which results in undesirable space-charge effects that greatly decrease the resolution due to electron scattering [34,35,49]. We do not include this effect in our numerics but acknowledge that it necessitates a compromise in the pulse lengths, which is reached with the given values of $\tau_{\mathrm{p}}$ and $\tau_{\mathrm{d}}$.

\section{NONEQUILIBRIUM ELECTRON DISTRIBUTION NEAR THE DIRAC POINT}

In Fig. 2(a), we show the electron distribution $n(\mathbf{k}=0, \omega)$ at the Dirac point at zero delay, i.e., $\Delta t=0$. We choose the driving frequency $\omega_{\mathrm{d}}=2 \pi \times 29.8 \mathrm{THz}$ and display the electron distribution as a function of the driving field strength
$E_{\mathrm{d}}$. We refer to the energy difference of the two distribution maxima that emerge at $\mathbf{k}=0$ as the energy gap $\Delta_{0}$. We see that this gap $\Delta_{0}$ grows monotonously as a function of $E_{\mathrm{d}}$ rather than being confined within the first Floquet zone of width $\omega_{\mathrm{d}}$, as also discussed in Ref. [50]. We derive the Floquet energy gap at the Dirac point from $h(\mathbf{k}=0, t)$ with a fixed vector potential amplitude $A_{\mathrm{d}}=E_{\mathrm{d}} / \omega_{\mathrm{d}}$. Using the Rabi solution, we obtain the energy (see Appendix B)

$$
\Delta_{0} / 2=\sqrt{\left(e v_{F} E_{\mathrm{d}} / \omega_{\mathrm{d}}\right)^{2}+\left(\hbar \omega_{\mathrm{d}} / 2\right)^{2}}-\hbar \omega_{\mathrm{d}} / 2 .
$$

In the following, we point out the most promising regime in which this gap can be detected. As we display in Fig. 2(a), the gap $\Delta_{0}$ grows with increasing driving strength $E_{\mathrm{d}}$; in particular it grows beyond the Floquet zone boundary at $\omega_{\mathrm{d}} / 2$. We propose to detect the energy gap $\Delta_{0}$ in this strongly driven regime in which $\hbar \omega_{\mathrm{d}} \approx \Delta_{0}$. While the Floquet quasienergies are confined to the Floquet zone, the maxima of the electron distribution continue to be shifted to higher frequencies with increasing $E_{\mathrm{d}}$ so that they can be resolved despite broadening effects and energy resolution limitations. For very large field strengths with $\Delta_{0} \gg \hbar \omega_{\mathrm{d}}$, the electrons will predominantly populate the lower bands at the Dirac point. Therefore, intermediate values of $E_{\mathrm{d}}$ are desirable, such that $\Delta_{0} \approx \hbar \omega_{\mathrm{d}}$, as we discuss throughout this paper.

In Fig. 2(b), we show the electron distribution $n(\mathbf{k}=$ $0, \omega)$ at zero delay, i.e., $\Delta t=0$, at the Dirac point for driving field strength $E_{\mathrm{d}}=20 \mathrm{MV} \mathrm{m}^{-1}$ as a function of the driving frequency $\omega_{\mathrm{d}}$. We display the energies $\pm \sqrt{\left(e v_{F} E_{\mathrm{d}} / \omega_{\mathrm{d}}\right)^{2}+\left(\hbar \omega_{\mathrm{d}} / 2\right)^{2}} \pm \hbar \omega_{\mathrm{d}} / 2$, which reproduce the 
maxima of the electron distribution. We see the expected scaling behavior of the gap at the Dirac point $\Delta_{0}$ of Eq. (12) as well as the spacing between the nearest Floquet replica $\Delta_{0}+\hbar \omega_{\mathrm{d}}$. We propose to measure the electron distribution in the regime that is given by $\omega_{\mathrm{d}}>\gamma$ and $\Delta_{0}>\hbar \gamma \cdot \gamma$ is given by $\gamma=\gamma_{ \pm} / 2+2 \gamma_{z}+\gamma_{\text {bg }}=75 \mathrm{THz} \approx 50 \mathrm{meV} / \hbar \approx$ $(13.3 \mathrm{fs})^{-1}$ as an overall metric for the decay rate. This value for $\gamma$ is comparable to the coherence times of $22 \mathrm{fs}$ found in Ref. [51]. With increasing $\omega_{\mathrm{d}}$ and for fixed $E_{\mathrm{d}}, \Delta_{0}$ decreases. This dependence is predicted by Eq. (12). If $\Delta_{0}$ is smaller than $\hbar \gamma$, the two maxima of the electron distribution are not resolved and are not detectable via trARPES. With decreasing $\omega_{\mathrm{d}}, \Delta_{0}$ increases and becomes easier to resolve. However, the driving period $2 \pi / \omega_{\mathrm{d}}$ needs to be shorter than the characteristic timescale of the dissipative processes, i.e., $\omega_{\mathrm{d}}>\gamma$. Otherwise, the picture of a close to adiabatically stirred Dirac cone in equilibrium is more appropriate than that of emerging Floquet-Bloch bands. Long scattering times have also been connected to the visibility of $\Delta_{0}$ in Ref. [52]. The range of feasible driving frequencies given by these two conditions decreases for increasing $\gamma$ but increases for increasing $E_{\mathrm{d}}$.

In general, the $m$-photon gaps $\Delta_{m}$ open up at momenta of $v_{F}|\mathbf{k}|=m \omega_{\mathrm{d}} / 2$, with $m>0$, for small field strengths $E_{\mathrm{d}}$. These gaps move inwards towards the Dirac point for increasing field strengths. Thus, increasing $E_{\mathrm{d}}$ increases the gap at the Dirac point but at the same time decreases the distance in momentum space to higher-order gaps. For the driving field strength [50]

$$
E_{\mathrm{d}}=\frac{\hbar \omega_{\mathrm{d}}^{2}}{e v_{F}} \sqrt{\frac{m^{2}}{4}+\frac{m}{2}}
$$

the $m$ th gap is located at $\mathbf{k}=0$ and merges with the gap $\Delta_{0}$. The next gap $\Delta_{m+1}$ is then the gap closest to the Dirac point with its distance maximized with respect to $E_{\mathrm{d}}$. This further enhances the visibility of the gap at the Dirac point and makes this relation between driving field strength and frequency desirable. We rewrite Eq. (13) to find the driving frequency that is necessary for a given field strength $E_{\mathrm{d}}$ to have the gap $\Delta_{0}$ be equal to $m$ times the driving frequency $\omega_{\mathrm{d}}$. It is

$$
\Omega_{m}=\left(\frac{m^{2}}{4}+\frac{m}{2}\right)^{-\frac{1}{4}} \sqrt{e v_{F} \hbar^{-1} E_{\mathrm{d}}} .
$$

The driving frequencies $\omega_{\mathrm{d}}=\Omega_{m}$ have the highest distinguishability and are indicated in Fig. 2(b) as vertical dashed lines. Additionally, at the frequency $\omega_{\mathrm{d}}=\Omega_{1}=$ $\left(\frac{4}{3}\right)^{\frac{1}{4}} \sqrt{e v_{F} \hbar^{-1} E_{\mathrm{d}}}$, the energy of the first Floquet replica at $\Delta_{0} / 2+\hbar \omega_{\mathrm{d}}$ is minimized. This point denotes a regime that is well suited for trARPES probing, and the conditions for resolvability simplify to $\gamma<\left(\frac{4}{3}\right)^{\frac{1}{4}} \sqrt{e v_{F} \hbar^{-1} E_{\mathrm{d}}}$. For $\gamma=75 \mathrm{THz}$ and $E_{\mathrm{d}} \approx 20 \mathrm{MV} \mathrm{m}^{-1}$, this suggests a driving frequency close to $\omega_{\mathrm{d}}=\Omega_{1} \approx 2 \pi \times 29.8 \mathrm{THz} \approx 123 \mathrm{meV}^{-1}$ or $\omega_{\mathrm{d}}=\Omega_{2} \approx$ $2 \pi \times 23 \mathrm{THz} \approx 96 \mathrm{meV}^{-1}$.

To demonstrate the steady state that emerges for driving at frequencies $\omega_{\mathrm{d}}=\Omega_{1}, \Omega_{2}$, and $\Omega_{3}$, we show the electron distribution $n(\mathbf{k}, \omega)$ in Fig. 3. This expands on the steadystate behavior of the electron distribution $n(\mathbf{k}=0, \omega)$ that we displayed in Fig. 2. We choose driving field strength $E_{\mathrm{d}}=20 \mathrm{MV} \mathrm{m}^{-1}$. In the vicinity of the Dirac point, band
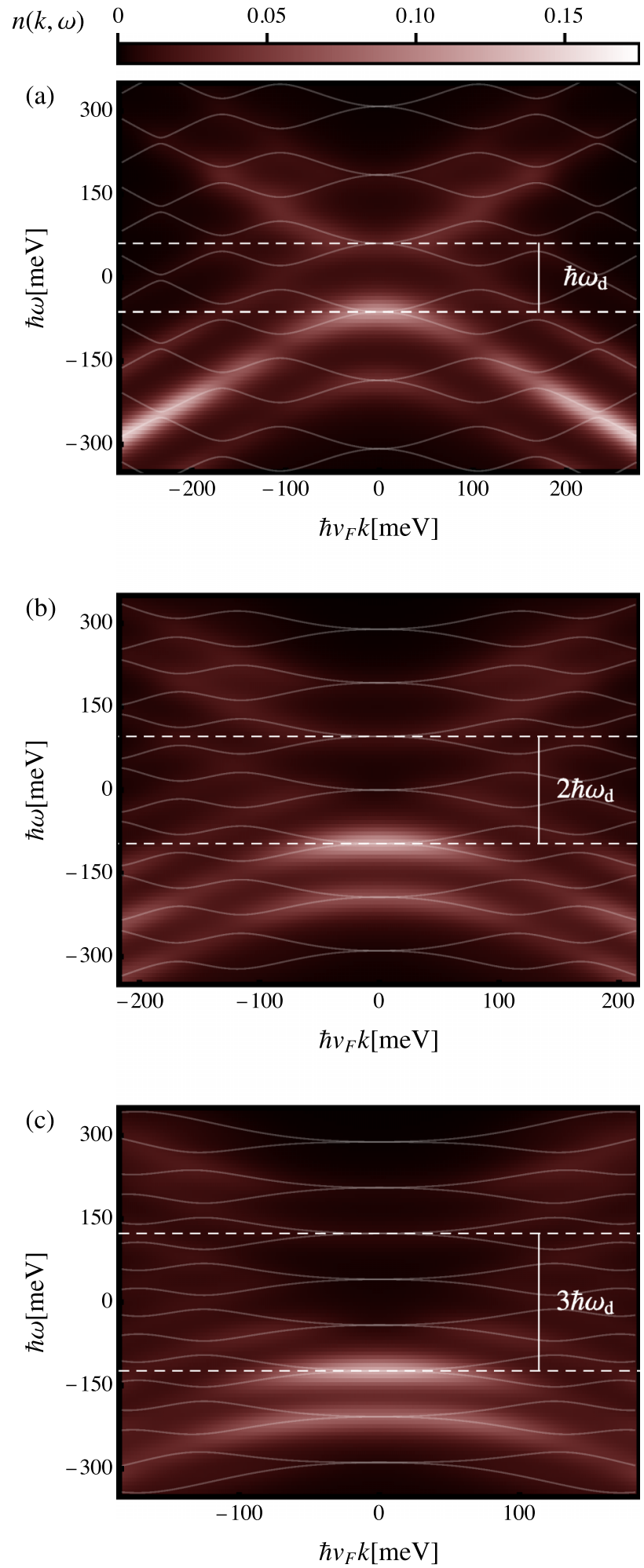

FIG. 3. The electron distribution $n(\mathbf{k}, \omega)$ for zero delay $(\Delta t=0)$ and driving field strength $E_{\mathrm{d}}=20 \mathrm{MV} \mathrm{m}^{-1}$. The driving frequencies are (a) $\omega_{\mathrm{d}}=\Omega_{1}$, (b) $\omega_{\mathrm{d}}=\Omega_{2}$, and (c) $\omega_{\mathrm{d}}=\Omega_{3}$. The dashed lines indicate an effective gap $\Delta_{0}$ of size $\hbar \omega_{\mathrm{d}}$ in (a), $2 \hbar \omega_{\mathrm{d}}$ in (b), and $3 \hbar \omega_{\mathrm{d}}$ in (c). The solid lines indicate the instantaneous spectra obtained from the Floquet Hamiltonian. 
occupations of the lower Floquet replicas are suppressed. The Floquet replicas with sizable electron occupation are two upper and two lower effective bands at the Dirac point, which are the four bands shown in Fig. 2. As the gap $\Delta_{0}$ increases, the population is predominantly distributed among the two lower bands. Away from the Dirac point, for nonzero k, the additional Floquet bands have sizable electron occupation and are visible in Fig. 3. The finite values of the electron distribution inside the Floquet gaps is a consequence of the broadening that occurs due to dissipative processes.

Having pointed out the regime that we propose to use to detect the energy gap at the Dirac point in terms of the driving field strength and the driving frequency, we now present the time-resolved response of the system. Figure 4 shows the electron distribution at the Dirac point $n(\mathbf{k}=0, \omega)$ as a function of the pulse delay time $\Delta t$ for the same driving field strength and driving frequencies as in Fig. 3, i.e., $\Omega_{1}, \Omega_{2}$, and $\Omega_{3}$ for $E_{\mathrm{d}}=20 \mathrm{MV} \mathrm{m}^{-1}$. This gives an estimate of the time-resolved Floquet-Bloch band occupations at the Dirac point. The dashed lines indicate the corresponding Floquet energies expected from static driving field strengths given by the drive pulse at the delay time $\Delta t$, i.e.,

$$
\begin{aligned}
\epsilon(\Delta t) & = \pm \sqrt{\exp \left\{-\frac{\Delta t^{2}}{\tau_{\mathrm{d}}^{2}} 8 \ln (2)\right\}\left(\frac{e v_{F} E_{\mathrm{d}}}{\omega_{\mathrm{d}}}\right)^{2}+\left(\frac{\hbar \omega_{\mathrm{d}}}{2}\right)^{2}} \\
& \pm \frac{\hbar \omega_{\mathrm{d}}}{2}
\end{aligned}
$$

The electron distributions that we show in Fig. 4 are close to the instantaneous steady-state distribution for this value of $\gamma$. Deviations from the instantaneous steady-state distribution manifest themselves as features that are asymmetric during the pulse rise and pulse decay. For this choice of $\gamma$ and of the pulse lengths, these features are small.

\section{LIMITATIONS OF RESOLUTION}

One common phenomenon that obscures the results of trARPES is LAPE [53]. The essentially free photoelectrons emitted in a trARPES experiment respond to the drive pulse with driving frequency $\omega_{\mathrm{d}}$. This may result in the photoelectron energy being shifted by one unit of the photon energy $\pm \hbar \omega_{\mathrm{d}}$. These energy shifts are detected in trARPES measurements as band replicas, whose similarities to Floquet replicas might hinder identifying the signatures of Floquet physics unambiguously. However, in contrast to Floquet replicas, these LAPE replicas are not related to band gaps [37]. The magnitude of the light-induced Floquet band gaps is tunable via the field strength $E_{\mathrm{d}}$ [see Eq. (12)]. We propose to use this tunability to distinguish the LAPE and Floquet replicas. More specifically, the Floquet replicas at the Dirac point are at $\pm \Delta_{0} / 2$ and $\pm\left(\Delta_{0} / 2+\hbar \omega_{\mathrm{d}}\right)$, as we show in Fig. 3 . The monotonous behavior of the Dirac gap $\Delta_{0}$ makes it possible to distinguish between LAPE and Floquet replicas.

The Floquet-Bloch bands resolved in $n(\mathbf{k}, \omega)$ are broadened due to dissipation. In addition they are Fourier broadened with the probe pulse length. The combined result is a Voigt
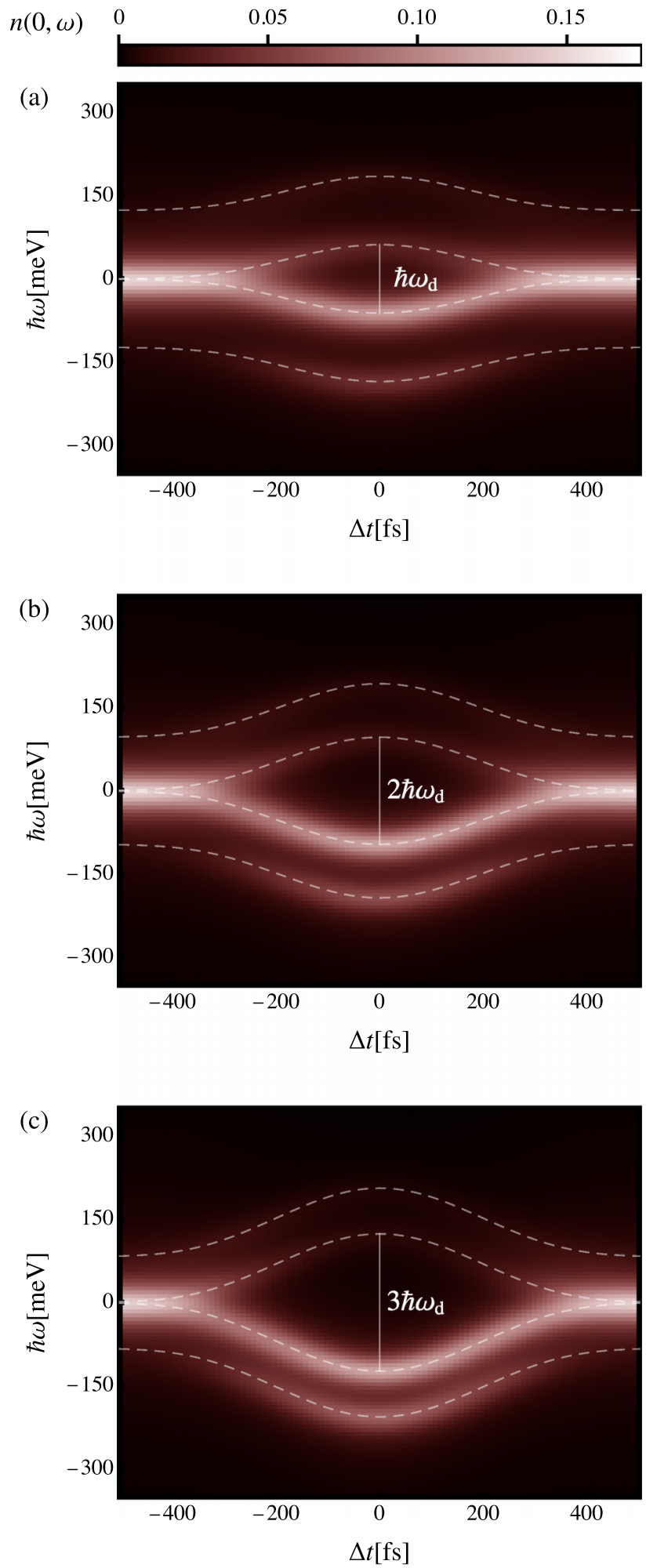

FIG. 4. The electron distribution $n(\mathbf{k}=0, \omega)$ at the Dirac point as a function of the delay time $\Delta t$ for a peak driving field strength of $E_{\mathrm{d}}=20 \mathrm{MV} \mathrm{m}^{-1}$. The driving frequencies are (a) $\omega_{\mathrm{d}}=\Omega_{1}$, (b) $\omega_{\mathrm{d}}=\Omega_{2}$, and (c) $\omega_{\mathrm{d}}=\Omega_{3}$. The dashed lines indicate the static Floquet energies corresponding to the driving field strengths at the center of the probe pulse. 
profile of approximate width

$$
\Gamma \approx \frac{\gamma}{2}+\sqrt{\frac{\gamma^{2}}{4}+\frac{4}{\tau_{\mathrm{p}}^{2}}},
$$

with $\gamma=\gamma_{ \pm} / 2+2 \gamma_{z}+\gamma_{\mathrm{bg}}$. For our specified values this is $\Gamma=80 \mathrm{THz}=(12.5 \mathrm{fs})^{-1} \approx 53 \mathrm{meV}^{-1}$. In order to successfully resolve the effective bands in $n(\mathbf{k}, \omega)$ it is crucial that the bands gaps are large compared to $\Gamma$. For probe pulses long enough that their contribution to $\Gamma$ can be neglected, the broadening is $\gamma$ due to intrinsic dissipation. Reducing $\gamma$ can be achieved by using cleaner graphene samples with higher mobility, which is technologically challenging.

Furthermore, trARPES experiments are, in general, limited by a Gaussian pulse energy resolution of the order [37]

$$
\Delta E \approx \tau_{\mathrm{p}}^{-1} 1825 \mathrm{meV} \text { fs. }
$$

The energy resolution of the measurement has to exceed the band gap $\Delta_{0}$, the Floquet replica spacing $\omega_{\mathrm{d}}$, and the Floquet-Bloch band Voigt width $\Gamma$. These requirements are realistically achieved in the proposed regime of $\Delta_{0}>\hbar \omega_{\mathrm{d}}$. For instance, fulfilling the resolvability conditions $\Gamma \ll \omega_{\mathrm{d}}$ and $\Delta E \ll \hbar \omega_{\mathrm{d}}$ is not a necessity for identifying signatures of Floquet physics for this regime.

To determine the minimal probe length that is necessary to achieve an energy resolution equal to the gap, we insert the expression for the gap $\Delta_{0}$ at the Dirac point into Eq. (17). It is

$$
\tau_{\mathrm{p}}^{\min }=\frac{1825 \mathrm{meV} \text { fs }}{\sqrt{4 e^{2} v_{F}^{2} E_{\mathrm{d}}^{2} / \omega_{\mathrm{d}}^{2}+\hbar^{2} \omega_{\mathrm{d}}^{2}}-\hbar \omega_{\mathrm{d}}} .
$$

An energy resolution several times better is necessary to clearly identify the Floquet-Bloch bands, which corresponds to probe pulse lengths several times larger than the minimal length, e.g., $\tau_{\mathrm{p}} \approx 10 \tau_{\mathrm{p}}^{\min }$.

\section{CONCLUSION}

In conclusion, we have pointed out a realistic regime for the detection of the light-induced topological gap in graphene via time- and angle-resolved photoelectron spectroscopy. Our proposed regime addresses the limitations of band broadening, energy and momentum resolution, and intrinsic limitations of the detection method for realistic estimates of dissipative processes. We find that these limitations are overcome by increasing the driving field strength and decreasing the driving frequency so that the energy difference between finitely occupied Floquet-Bloch bands is larger than the Floquet zone. The timescales associated with the dissipative processes set the limits of this regime. On the one hand, the driving frequency has to be large enough that many driving cycles occur during one characteristic timescale of the dissipation. On the other, decreasing the driving frequency increases the gap size at the Dirac point, which has to exceed the band broadening. As the gap becomes larger than multiples of the driving frequency, limitations such as band broadening and inherent energy resolutions no longer obstruct the identifica- tion of signatures of Floquet physics. This regime also allows undesired laser-assisted photoemission replicas in trARPES measurements to be unambiguously identified as such at the Dirac point and to be clearly distinguished from the Floquet replicas. The detection of Floquet bands via trARPES would constitute a profound insight in light-driven solids, which would complement the measurements of transport reported in Ref. [8] and thereby advance the field of optical control of solids.

\section{ACKNOWLEDGMENTS}

We thank K. Rossnagel, J. McIver, and G. Jotzu for very insightful discussions. This work is funded by the Deutsche Forschungsgemeinschaft (DFG, German Research Foundation), SFB-925, Project No. 170620586, and the Cluster of Excellence "Advanced Imaging of Matter" (EXC 2056), Project No. 390715994.

\section{APPENDIX A: MASTER EQUATION}

We base our numerics on the Lindblad-von Neumann master equation

$$
\dot{\rho}=i[\rho, H]+\sum_{j} c_{j}\left(L_{j} \rho L_{j}^{\dagger}-\frac{1}{2}\left\{L_{j}^{\dagger} L_{j}, \rho\right\}\right),
$$

where $j \in\{z,+,-, \pm 1, \pm 2, \pm 3, \pm 4\}$. We consider a product state in momentum space $\rho=\prod_{k} \rho_{k}$ and the Hamiltonian in Eq. (3) in the extended basis spanned by the states $|0\rangle$, $c_{\mathbf{k}, \mathrm{A}}^{\dagger}|0\rangle, c_{\mathbf{k}, \mathrm{B}}^{\dagger}|0\rangle$, and $c_{\mathbf{k}, \mathrm{B}}^{\dagger} c_{\mathbf{k}, \mathrm{A}}^{\dagger}|0\rangle$. The transformation $V$ into the instantaneous eigenbasis diagonalizes the Hamiltonian at any given time and determines the momentum-dependent Lindblad operators as

$$
\begin{gathered}
L_{z}=V\left(c_{\mathbf{k}, \mathrm{A}}^{\dagger} c_{\mathbf{k}, \mathrm{A}}-c_{\mathbf{k}, \mathrm{B}}^{\dagger} c_{\mathbf{k}, \mathrm{B}}\right) V^{\dagger}, \\
L_{+}=V c_{\mathbf{k}, \mathrm{B}}^{\dagger} c_{\mathbf{k}, \mathrm{A}} V^{\dagger}, \\
L_{-}=V c_{\mathbf{k}, \mathrm{A}}^{\dagger} c_{\mathbf{k}, \mathrm{B}} V^{\dagger}, \\
L_{l}=V\left(\begin{array}{cccc}
0 & \delta_{-1, l} & \delta_{-2, l} & 0 \\
\delta_{1, l} & 0 & 0 & \delta_{-3, l} \\
\delta_{2, l} & 0 & 0 & \delta_{-4, l} \\
0 & \delta_{3, l} & \delta_{4, l} & 0
\end{array}\right) V^{\dagger} .
\end{gathered}
$$

The coefficients $c_{j}$ are

$$
\begin{gathered}
c_{z}=\gamma_{z}, \\
c_{+}+c_{-}=\gamma_{ \pm}, \quad c_{+}=c_{-} e^{-2 \epsilon \beta}, \\
c_{j}+c_{-j}=\gamma_{\text {bg }}, \quad c_{-1,2,3,-4}=c_{1,-2,-3,4} e^{\epsilon \beta},
\end{gathered}
$$

with $\beta=\left(k_{\mathrm{B}} T\right)^{-1}$ and the instantaneous level spacing $\epsilon$. This four-dimensional description makes two-point correlation functions and therefore the electron distributions via Eqs. (8) and (9) accessible. 


\section{APPENDIX B: FLOQUET ENERGIES}

At the Dirac point, the graphene Hamiltonian in Eq. (3) with constant amplitude simplifies to

$$
h(\mathbf{k}=0, t)=\frac{e v_{F}}{\hbar} A_{\mathrm{d}}\left[\sin \left(\omega_{\mathrm{d}} t\right) \sigma_{x}+\cos \left(\omega_{\mathrm{d}} t\right) \sigma_{y}\right],
$$

which is the Rabi-Hamiltonian

$$
H_{\mathrm{R}}=\hbar\left(\begin{array}{cc}
\omega_{0} / 2 & \Omega e^{-i \omega_{\mathrm{d}} t} \\
\Omega e^{+i \omega_{\mathrm{d}} t} & -\omega_{0} / 2
\end{array}\right),
$$

with $\omega_{0}=0$. In the rotating frame given by $\exp \left\{-i \sigma_{z} \omega_{\mathrm{d}} t / 2\right\}$, the corresponding dynamics are governed by the static Hamiltonian

$$
H_{\mathrm{R}}^{\prime}=\hbar\left(\begin{array}{cc}
-\omega_{\mathrm{d}} / 2 & \Omega \\
\Omega & +\omega_{\mathrm{d}} / 2
\end{array}\right)
$$

with eigenenergies

$$
\epsilon=\hbar \sqrt{\Omega^{2}+\omega_{\mathrm{d}}^{2} / 4}
$$

In the original frame of Eq. (B2) the solutions to the Schrödinger equation then rotate with the energies

$$
\epsilon= \pm \hbar\left(\sqrt{\Omega^{2}+\omega_{\mathrm{d}}^{2} / 4}-\omega_{\mathrm{d}} / 2\right)
$$

which gives Eq. (12) for $\Omega=e v_{F} \hbar^{-1} E_{\mathrm{d}} / \omega_{\mathrm{d}}$.

This expression increases monotonously as a function of $E_{\mathrm{d}}$, and the Floquet energies are given by this expression modulo the driving frequency $\omega_{\mathrm{d}}$. Put differently, $\epsilon \pm m \hbar \omega_{\mathrm{d}}$ gives the various Floquet replicas at the Dirac point. Whenever $\epsilon=m \hbar \omega_{\mathrm{d}} / 2$, the expression of $\epsilon$ in Eq. (B5) crosses a Floquet zone boundary. We solve this condition for $E_{\mathrm{d}}$ to find the driving field strengths at which this occurs for the $m$ th Floquet zone boundary. This gives us Eq. (13).

In the case of a time-dependent driving amplitude such as in Eq. (7) and under the assumption that the Floquet states form instantly, we insert the driving amplitude envelope at a given point in time $\Delta t$ expressed relative to the amplitude peak, such that

$$
\Omega(\Delta t)=\frac{e v_{F}}{\hbar} \frac{E_{\mathrm{d}}}{\omega_{\mathrm{d}}} \exp \left\{-(\Delta t)^{2} \tau_{\mathrm{d}}^{-2} 4 \ln (2)\right\} .
$$

This leads to the expression $\epsilon(\Delta t)$ in Eq. (15).
[1] D. N. Basov, R. D. Averitt, and D. Hsieh, Towards properties on demand in quantum materials, Nat. Mater. 16, 1077 (2017).

[2] N. H. Lindner, G. Refael, and V. Galitski, Floquet topological insulator in semiconductor quantum wells, Nat. Phys. 7, 490 (2011).

[3] T. Kitagawa, T. Oka, A. Brataas, L. Fu, and E. Demler, Transport properties of nonequilibrium systems under the application of light: Photoinduced quantum hall insulators without landau levels, Phys. Rev. B 84, 235108 (2011).

[4] M. Bukov, L. D’Alessio, and A. Polkovnikov, Universal high-frequency behavior of periodically driven systems: From dynamical stabilization to Floquet engineering, Adv. Phys. 64, 139 (2015)

[5] T. Oka and H. Aoki, Photovoltaic Hall effect in graphene, Phys. Rev. B 79, 081406(R) (2009).

[6] G. Usaj, P. M. Perez-Piskunow, L. E. F. Foa Torres, and C. A. Balseiro, Irradiated graphene as a tunable Floquet topological insulator, Phys. Rev. B 90, 115423 (2014).

[7] M. Vogl, M. Rodriguez-Vega, and G. A. Fiete, Effective Floquet Hamiltonian in the low-frequency regime, Phys. Rev. B 101, 024303 (2020).

[8] J. W. McIver, B. Schulte, F.-U. Stein, T. Matsuyama, G. Jotzu, G. Meier, and A. Cavalleri, Light-induced anomalous Hall effect in graphene, Nat. Phys. 16, 38 (2019).

[9] M. Nuske, L. Broers, B. Schulte, G. Jotzu, S. A. Sato, A. Cavalleri, A. Rubio, J. W. McIver, and L. Mathey, Floquet dynamics in light-driven solids, Phys. Rev. Res. 2, 043408 (2020).

[10] D. Kutnyakhov et al., Time- and momentum-resolved photoemission studies using time-of-flight momentum microscopy at a free-electron laser, Rev. Sci. Instrum. 91, 013109 (2020).

[11] C. M. Cacho, I. C. E. Turcu, C. A. Froud, W. A. Bryan, G. R. A. J. Nemeth, J. C. Petersen, N. Dean, A. Cavalleri, S.
Kaiser, A. Simoncig, H. Y. Liu, A. L. Cavalieri, S. Dhesi, L. Poletto, P. Villoresi, F. Frassetto, and E. Springate, Ultrafast tr-ARPES with Artemis xuv beamline, in Research in Optical Sciences (Optical Society of America, Washington, DC, 2012), p. JT2A.43.

[12] S. Peli, D. Puntel, D. Kopic, B. Sockol, F. Parmigiani, and F. Cilento, Time-resolved vuv ARPES at $10.8 \mathrm{eV}$ photon energy and $\mathrm{mHz}$ repetition rate, J. Electron Spectrosc. Relat. Phenom. 243, 146978 (2020).

[13] G. Rohde, A. Hendel, A. Stange, K. Hanff, L.-P. Oloff, L. X. Yang, K. Rossnagel, and M. Bauer, Time-resolved ARPES with sub-15 fs temporal and near Fourier-limited spectral resolution, Rev. Sci. Instrum. 87, 103102 (2016).

[14] S. Eich, A. Stange, A. V. Carr, J. Urbancic, T. Popmintchev, M. Wiesenmayer, K. Jansen, A. Ruffing, S. Jakobs, T. Rohwer, S. Hellmann, C. Chen, P. Matyba, L. Kipp, K. Rossnagel, M. Bauer, M. M. Murnane, H. C. Kapteyn, S. Mathias, and M. Aeschlimann, Time- and angle-resolved photoemission spectroscopy with optimized high-harmonic pulses using frequency-doubled Ti:sapphire lasers, J. Electron Spectrosc. Relat. Phenom. 195, 231 (2014).

[15] M. Puppin, Y. Deng, C. W. Nicholson, J. Feldl, N. B. M Schröter, H. Vita, P. S. Kirchmann, C. Monney, L. Rettig, M. Wolf, and R. Ernstorfer, Time- and angle-resolved photoemission spectroscopy of solids in the extreme ultraviolet at $500 \mathrm{kHz}$ repetition rate, Rev. Sci. Instrum. 90, 023104 (2019).

[16] C. W. Nicholson, A. Lücke, W. G. Schmidt, M. Puppin, L. Rettig, R. Ernstorfer, and M. Wolf, Beyond the molecular movie: Dynamics of bands and bonds during a photoinduced phase transition, Science 362, 821 (2018).

[17] I. Gierz, S. Link, U. Starke, and A. Cavalleri, Non-equilibrium Dirac carrier dynamics in graphene investigated with time- and 
angle-resolved photoemission spectroscopy, Faraday Discuss. 171, 311 (2014).

[18] S. Ulstrup, J. C. Johannsen, A. Crepaldi, F. Cilento, M. Zacchigna, C. Cacho, R. T. Chapman, E. Springate, F. Fromm, C. Raidel, T. Seyller, F. Parmigiani, M. Grioni, and P. Hofmann, Ultrafast electron dynamics in epitaxial graphene investigated with time- and angle-resolved photoemission spectroscopy, J. Phys.: Condens. Matter 27, 164206 (2015).

[19] I. Gierz, Probing carrier dynamics in photo-excited graphene with time-resolved ARPES, J. Electron Spectrosc. Relat. Phenom. 219, 53 (2017).

[20] S. Aeschlimann, A. Rossi, M. Chávez-Cervantes, R. Krause, B. Arnoldi, B. Stadtmüller, M. Aeschlimann, S. Forti, F. Fabbri, C. Coletti, and I. Gierz, Direct evidence for efficient ultrafast charge separation in epitaxial $\mathrm{WS}_{2}$ /graphene heterostructures, Sci. Adv. 6, 20 (2020).

[21] R. Krause, M. Chávez-Cervantes, S. Aeschlimann, S. Forti, F. Fabbri, A. Rossi, C. Coletti, C. Cacho, Y. Zhang, P. E. Majchrzak, R. T. Chapman, E. Springate, and I. Gierz, Ultrafast charge separation in bilayer $\mathrm{WS}_{2}$ /graphene heterostructure revealed by time- and angle-resolved photoemission spectroscopy, Front. Phys. 9, 184 (2021).

[22] S. Dong, M. Puppin, T. Pincelli, S. Beaulieu, D. Christiansen, H. Hübener, C. W. Nicholson, R. P. Xian, M. Dendzik, Y. Deng, Y. W. Windsor, M. Selig, E. Malic, A. Rubio, A. Knorr, M. Wolf, L. Rettig, and R. Ernstorfer, Direct measurement of key exciton properties: Energy, dynamics, and spatial distribution of the wave function, Nat. Sci. 1, e10010 (2021).

[23] J. Maklar, S. Dong, S. Beaulieu, T. Pincelli, M. Dendzik, Y. W. Windsor, R. P. Xian, M. Wolf, R. Ernstorfer, and L. Rettig, A quantitative comparison of time-of-flight momentum microscopes and hemispherical analyzers for time- and angleresolved photoemission spectroscopy experiments, Rev. Sci. Instrum. 91, 123112 (2020).

[24] R. Liu, Y. Ogawa, P. Chen, K. Ozawa, T. Suzuki, M. Okada, T. Someya, Y. Ishida, K. Okazaki, S. Shin, T. Chiang, and I. Matsuda, Femtosecond to picosecond transient effects in $\mathrm{WSe}_{2}$ observed by pump-probe angle-resolved photoemission spectroscopy, Sci. Rep. 7, 15981 (2017).

[25] I. Gierz, M. Mitrano, J. C. Petersen, C. Cacho, I. C. E. Turcu, E. Springate, A. Stöhr, A. Köhler, U. Starke, and A. Cavalleri, Population inversion in monolayer and bilayer graphene, J. Phys.: Condens. Matter 27, 164204 (2015).

[26] F. Mahmood, C. Chan, Z. Alpichshev, D. Gardner, Y. Lee, P. A. Lee, and N. Gedik, Selective scattering between Floquet-Bloch and Volkov states in a topological insulator, Nat. Phys. 12, 306 (2016).

[27] H. Soifer, A. Gauthier, A. F. Kemper, C. R. Rotundu, S.-L. Yang, H. Xiong, D. Lu, M. Hashimoto, P. S. Kirchmann, J. A. Sobota, and Z.-X. Shen, Band-Resolved Imaging of Photocurrent in a Topological Insulator, Phys. Rev. Lett. 122, 167401 (2019).

[28] Y. H. Wang, D. Hsieh, D. Pilon, L. Fu, D. R. Gardner, Y. S. Lee, and N. Gedik, Observation of a Warped Helical Spin Texture in $\mathrm{Bi}_{2} \mathrm{Se}_{3}$ from Circular Dichroism Angle-Resolved Photoemission Spectroscopy, Phys. Rev. Lett. 107, 207602 (2011).

[29] M. A. Sentef, M. Claassen, A. F. Kemper, B. Moritz, T. Oka, J. K. Freericks, and T. P. Devereaux, Theory of Floquet band formation and local pseudospin textures in pump-probe photoemission of graphene, Nat. Commun. 6, 7047 (2015).

[30] L. P. Gavensky, G. Usaj, and C. A. Balseiro, Photo-electrons unveil topological transitions in graphene-like systems, Sci. Rep. 6, 36577 (2016).

[31] M. Schüler, U. De Giovannini, H. Hübener, A. Rubio, M. A. Sentef, T. P. Devereaux, and P. Werner, How Circular Dichroism in Time- and Angle-Resolved Photoemission Can Be Used to Spectroscopically Detect Transient Topological States in Graphene, Phys. Rev. X 10, 041013 (2020).

[32] S. Beaulieu, J. Schusser, S. Dong, M. Schüler, T. Pincelli, M. Dendzik, J. Maklar, A. Neef, H. Ebert, K. Hricovini, M. Wolf, J. Braun, L. Rettig, J. Minár, and R. Ernstorfer, Revealing Hidden Orbital Pseudospin Texture with Time-Reversal Dichroism in Photoelectron Angular Distributions, Phys. Rev. Lett. 125, 216404 (2020).

[33] Y. H. Wang, H. Steinberg, P. Jarillo-Herrero, and N. Gedik, Observation of Floquet-Bloch states on the surface of a topological insulator, Science 342, 453 (2013).

[34] S. Hellmann, T. Ott, L. Kipp, and K. Rossnagel, Vacuum spacecharge effects in nano-ARPES, Phys. Rev. B 85, 075109 (2012).

[35] S. Hellmann, K. Rossnagel, M. Marczynski-Bühlow, and L. Kipp, Vacuum space-charge effects in solid-state photoemission, Phys. Rev. B 79, 035402 (2009).

[36] L.-P. Oloff, K. Hanff, A. Stange, G. Rohde, F. Diekmann, M. Bauer, and K. Rossnagel, Pump laser-induced spacecharge effects in HHG-driven time- and angle-resolved photoelectron spectroscopy, J. Appl. Phys. 119, 225106 (2016).

[37] B. Lv, T. Qian, and H. Ding, Angle-resolved photoemission spectroscopy and its application to topological materials, Nat. Rev. Phys. 1, 609 (2019).

[38] T. O. Wehling, A. V. Balatsky, M. I. Katsnelson, A. I. Lichtenstein, K. Scharnberg, and R. Wiesendanger, Local electronic signatures of impurity states in graphene, Phys. Rev. B 75, 125425 (2007).

[39] S. Ihnatsenka and G. Kirczenow, Dirac point resonances due to atoms and molecules adsorbed on graphene and transport gaps and conductance quantization in graphene nanoribbons with covalently bonded adsorbates, Phys. Rev. B 83, 245442 (2011).

[40] M. Inglot and V. K. Dugaev, Impurity states in graphene with intrinsic spin-orbit interaction, J. Appl. Phys. 109, 123709 (2011).

[41] Y. G. Pogorelov, V. M. Loktev, and D. Kochan, Impurity resonance effects in graphene versus impurity location, concentration, and sublattice occupation, Phys. Rev. B 102, 155414 (2020).

[42] T. Higuchi, C. Heide, K. Ullmann, H. B. Weber, and P. Hommelhoff, Light-field-driven currents in graphene, Nature (London) 550, 224 (2017).

[43] I. Gierz, F. Calegari, S. Aeschlimann, M. Chávez Cervantes, C. Cacho, R. T. Chapman, E. Springate, S. Link, U. Starke, C. R. Ast, and A. Cavalleri, Tracking Primary Thermalization Events in Graphene with Photoemission at Extreme Time Scales, Phys. Rev. Lett. 115, 086803 (2015).

[44] M. Breusing, C. Ropers, and T. Elsaesser, Ultrafast Carrier Dynamics in Graphite, Phys. Rev. Lett. 102, 086809 (2009). 
[45] I. Gierz, J. C. Petersen, M. Mitrano, C. Cacho, I. C. E. Turcu, E. Springate, A. Stöhr, A. Köhler, U. Starke, and A. Cavalleri, Snapshots of non-equilibrium Dirac carrier distributions in graphene, Nat. Mater. 12, 1119 (2013).

[46] M. Breusing, S. Kuehn, T. Winzer, E. Malić, F. Milde, N. Severin, J. P. Rabe, C. Ropers, A. Knorr, and T. Elsaesser, Ultrafast nonequilibrium carrier dynamics in a single graphene layer, Phys. Rev. B 83, 153410 (2011).

[47] J. K. Freericks, H. R. Krishnamurthy, and Th. Pruschke, Theoretical Description of Time-Resolved Photoemission Spectroscopy: Application to Pump-Probe Experiments, Phys. Rev. Lett. 102, 136401 (2009).

[48] This expression for $\mathcal{G}\left(k, t_{2}, t_{1}\right)$ omits coherence terms such as $c_{k, A}^{\dagger}\left(t_{2}\right) c_{k, B}\left(t_{1}\right)$, which are responsible for the dark corridor in graphene.

[49] J. Graf, S. Hellmann, C. Jozwiak, C. L. Smallwood, Z. Hussain, R. A. Kaindl, L. Kipp, K. Rossnagel, and A. Lanzara, Vacuum space charge effect in laser-based solid-state photoemission spectroscopy, J. Appl. Phys. 107, 014912 (2010).

[50] L. Broers and L. Mathey, Observing light-induced Floquet band gaps in the longitudinal conductivity of graphene, Comm. Phys. 4, 248 (2021).

[51] C. Heide, T. Eckstein, T. Boolakee, C. Gerner, H. B. Weber, I. Franco, and P. Hommelhoff, Electronic coherence and coherent dephasing in the optical control of electrons in graphene, Nano Lett. 21, 9403 (2021).

[52] S. Aeschlimann, S. A. Sato, R. Krause, M. Chávez-Cervantes, U. De Giovannini, H. Hübener, S. Forti, C. Coletti, K. Hanff, K. Rossnagel, A. Rubio, and I. Gierz, Survival of Floquet-Bloch states in the presence of scattering, Nano Lett. 21, 5028 (2021).

[53] G. Saathoff, L. Miaja-Avila, M. Aeschlimann, M. M. Murnane, and H. C. Kapteyn, Laser-assisted photoemission from surfaces, Phys. Rev. A 77, 022903 (2008). 